Artículo científico

Volumen 32(1): 137-148. Enero-abril, 2021

e-ISSN 2215-3608, doi:10.15517/am.v32i1.39822

https://www.revistas.ucr.ac.cr/index.php/agromeso

\title{
Fertilización con nitrógeno y potasio en maíz en un Alfisol de Guanacaste, Costa Rica ${ }^{1}$
}

\section{Nitrogen and potassium fertilization in corn in an Alfisol of Guanacaste, Costa Rica}

\author{
Gabriel Garbanzo-León ${ }^{2}$, Alfredo Alvarado-Hernández², Jorge Claudio Vargas-Rojas ${ }^{3}$, \\ Gilberto Cabalceta-Aguilar ${ }^{2}$ Edgar V. Vega-Villalobos ${ }^{4}$
}

1 Recepción: 10 de diciembre, 2019. Aceptación: 11 de junio, 2020. Este trabajo formó parte del proyecto de investigación VI 733 B7 290 inscrito en Vicerrectoría de Investigación de la Universidad de Costa Rica, San José, Costa Rica.

2 Universidad de Costa Rica, Escuela de Agronomía, Centro de Investigaciones Agronómicas, Sabanilla, San José. C.P. 11501, San José, Costa Rica. juan.garbanzo@ucr.ac.cr (autor para la correspondencia; https://orcid.org/0000-0003-2848-6199), alfredo.alvarado@ucr.ac.cr (https:// orcid.org/0000-0002-6930-6660), gilberto@cabalceta.com (https://orcid.org/0000-0002-3757-8207).

3 Universidad de Costa Rica, Carrera de Agronomía, Finca Experimental de Santa Cruz, Sede de Guanacaste, Costa Rica. jorgeclaudio. vargas@ucr.ac.cr (https://orcid.org/0000-0002-1139-2148).

4 Universidad de Costa Rica, Escuela de Agronomía, Departamento de Cultivos, San José, Costa Rica. edgar.vega@ucr.ac.cr (https://orcid. org/0000-0002-5678-1710).

\section{Resumen}

Introducción. En Guanacaste, Costa Rica, el maíz (Zea mays) es un cultivo de consumo cultural desde épocas precolombinas, muchos agricultores destinan zonas para su siembra en asocio con otros cultivos. Sin embargo, las variedades utilizadas requieren estudios de fertilización y nutrición para mejorar la calidad de las mazorcas y vida postcosecha. Objetivo. Evaluar en la variedad de maíz J-Sáenz, el efecto de dosis crecientes de nitrógeno y potasio, en combinación con tres densidades de siembra. Materiales y métodos. El estudio se realizó en la Finca Experimental de Santa Cruz, Universidad de Costa Rica, en 2018. Se evaluaron tres dosis de N (100, 200, $300 \mathrm{~kg} \mathrm{ha}^{-1}$ con una base de $160 \mathrm{~kg} \mathrm{ha}^{-1}$ de $\left.\mathrm{K}_{2} \mathrm{O}\right)$ y $\mathrm{K}_{2} \mathrm{O}\left(100,160\right.$ y $200 \mathrm{~kg} \mathrm{ha}^{-1}$ con una base de $\left.300 \mathrm{~kg} \mathrm{~N} \mathrm{ha}^{-1}\right)$ con tres densidades de siembra (50 000, 57143 y 66667 plantas ha-1) con la variedad J-Sáenz. Se evaluó el rendimiento del cultivo, el porcentaje de mazorca expuesta y la acumulación de nutrimentos en estado fenológico R5. Los datos se sometieron a análisis de varianza con estructura factorial y modelos lineales generalizados con pruebas de separación de medias DGC. Resultados. Hubo incremento significativo en la producción de grano al aumentar la densidad de siembra de 50000 a 66667 plantas ha ${ }^{-1}$. La mayor eficiencia en el uso de N y K se logró con la aplicación de 100 kg de cada elemento a la densidad de 57000 plantas ha-1. La variable mazorca expuesta no se relacionó con las dosis de nutrimentos. El maíz aumentó la absorción con el incremento de las dosis de $\mathrm{N}$ pero no de K. Conclusión. La densidad de siembra de 57143 plantas ha-1 mostró el mejor rendimiento a dosis de $100 \mathrm{~kg} \mathrm{de} \mathrm{N} \mathrm{ha}^{-1}$ y $100 \mathrm{~kg}$ de $\mathrm{K}_{2} \mathrm{O}_{\text {ha }}{ }^{-1}$.

Palabras clave: absorción de nutrimentos, trópico, nutrición vegetal, densidad de planta.

\section{Abstract}

Introduction. In Guanacaste, Costa Rica, corn (Zea mays) has been a cultural crop for consumption since preColombian times. Many farmers have set aside areas for its planting in association with other crops. However, the 
varieties used require fertilization and plant nutrition studies to improve ear quality and postharvest life. Objective. To evaluate the effect of increasing doses of nitrogen and potassium in the J-Saenz maize variety, in combination with three sowing densities. Materials and methods. The study was conducted at the Santa Cruz Experimental Farm, Universidad de Costa Rica, in 2018. Three doses of N (100, 200, $300 \mathrm{~kg} \mathrm{ha}^{-1}$ with a base of $160 \mathrm{~kg} \mathrm{ha}^{-1}$ of $\left.\mathrm{K}_{2} \mathrm{O}\right)$ and $\mathrm{K}_{2} \mathrm{O}\left(100,160\right.$ and $200 \mathrm{~kg} \mathrm{ha}^{-1}$ with a base of $\left.300 \mathrm{~kg} \mathrm{~N} \mathrm{ha}^{-1}\right)$ were evaluated with three sowing densities (50 000, 57 143, and 66667 plants ha ${ }^{-1}$ ) with the J-Sáenz variety. The crop yield, the percentage of exposed ear, and the accumulation of nutrients in the phenological R5 stage were evaluated. The data were subjected to analysis of variance with a factorial structure and generalized linear models with DGS tests of means separation. Results. There was a significant increase in grain production by increasing the sowing density from 50000 to 66667 plants ha $^{-1}$. The highest efficiency in the $\mathrm{N}$ and $\mathrm{K}$ use was achieved with the application of $100 \mathrm{~kg} \mathrm{ha}^{-1}$ of each element at a density of 57 000 plants ha ${ }^{-1}$. The exposed ear variable was not related to nutrient doses. Corn increased absorption with increasing doses of $\mathrm{N}$ but not K. Conclusion. The planting density of 57143 plants ha ${ }^{-1}$ showed the best yield at doses of 100 $\mathrm{kg}$ of $\mathrm{N} \mathrm{ha}^{-1}$ and $100 \mathrm{~kg}$ of $\mathrm{K}_{2} \mathrm{O} \mathrm{ha}{ }^{-1}$.

Keywords: nutrients uptake, tropic, plant nutrition, plant density.

\section{Introducción}

El maíz (Zea mayz) es el tercer cultivo en importancia a nivel mundial y uno de los de mayor consumo en Centro América. En Costa Rica se cultiva desde la época precolombina y es utilizado por los pequeños productores de las zonas rurales para consumo de grano y harina (p.e. tortillas y tamales), la fabricación de bebidas (p.e. chicha), la alimentación de animales y para la venta local de excedentes (Sáenz, 1970). Los estudios sobre el cultivo del maíz en Costa Rica presentan gran diversidad en sus recomendaciones de manejo (Salas, 1971; Salas \& Bonilla, 1961), debido a la gran cantidad de variedades de libre polinización y materiales criollos que se encuentran en manos de productores, muchos de estos seleccionados por ellos mismos para la producción en una zona específica (Aguilar et al., 2015; Bonilla \& Meléndez, 2005). En la provincia de Guanacaste, el maíz se siembra en los meses de junio hasta octubre para aprovechar los meses de mayor precipitación y garantizar la producción, hoy en día se emplean variedades mejoradas con tolerancia a la sequía, adaptadas a temperaturas elevadas y con una productividad aceptable (Bonilla et al., 2008).

La mayoría de los cereales como el maíz responden a la fertilización, en particular de N, P y K. La respuesta al $\mathrm{N}$ varía entre 60 y $150 \mathrm{~kg} \mathrm{ha}^{-1}$ en Ultisoles y Oxisoles trabajados de forma intensiva, es casi nula en terrenos recién desmontados de suelos ácidos y bajos en P (Bartholomew, 1973). En las tierras altas de Centroamérica y México la respuesta a la aplicación de $\mathrm{N}$ es común y cuando no ocurre se atribuye a efectos de la sequía, mal control de malezas, anegamiento y elevados niveles de acidez en el suelo (Bartholomew, 1973; Alvarado et al., 2001). En el Arco Seco de Panamá, Gordon et al. (2000) observaron respuesta significativa a la adición fraccionada de $120 \mathrm{~kg}$ N como urea en diez de doce localizadas comparadas, lográndose elevar el rendimiento y la eficiencia de uso del $\mathrm{N}$ con la adición de $10 \%$ del fertilizante a la siembra y entre 40 y $50 \%$ a los 21 y 40 días después de la siembra. Al evaluar en maíz híbrido NH-INTA-991 las densidades de siembra de 35 000, 50000 y 62500 plantas ha ${ }^{-1}$ y la adición de $0,75,150$ y $225 \mathrm{~kg} \mathrm{~N} \mathrm{ha}^{-1}$ como urea en Masatepe (Masaya, Nicaragua), Castillo \& Bird (2013) determinaron que el factor densidad mostró efecto significativo sobre la altura y diámetro de la planta y el rendimiento de grano. Los niveles de nitrógeno y las densidades de 50000 y 62500 plantas ha $^{-1}$ presentaron respuesta lineal y cuadrática, respectivamente. La mejor relación beneficio-costo se obtuvo al hacer uso de la dosis de $75 \mathrm{~kg} \mathrm{ha}^{-1}$ con las densidades poblacionales de 50000 y 62500 plantas ha $^{-1}$.

La respuesta a la adición de $\mathrm{P}\left(80-150 \mathrm{~kg}_{\text {de }} \mathrm{P}_{2} \mathrm{O}_{5}\right.$ ha $\left.^{-1}\right)$ es común en suelos rojos, ácidos y arcillosos de América Latina, ha permitido incrementos de rendimiento de hasta el $100 \%$, así como en suelos derivados de ceniza volcánica 
donde los rendimientos aumentan en más de un $25 \%\left(1,15\right.$ a 2,17 t ha-1) con la adición de $40 \mathrm{~kg}$ de $\mathrm{P}_{2} \mathrm{O}_{5}$ ha ${ }^{-1}$ (Bartholomew, 1973). Al estudiar el efecto a mediano y largo plazo de la adición de 0, 25, 50, 75 y $100 \mathrm{~kg} \mathrm{P}^{-1}$ al cultivo de maíz en Udic Haplustalfs del Arco Seco de Panamá y adiciones en banda por diez años sucesivos de una base de $\mathrm{N}$ y K con 0,10, 20 and $30 \mathrm{~kg} \mathrm{P} \mathrm{ha}^{-1}$, Gordon-Mendoza et al. (2016) encontraron que el rendimiento máximo de grano se logró con la adición de $304 \mathrm{~kg}$ de $\mathrm{P}$ (promedio $30,4 \mathrm{~kg} \mathrm{P}^{-1}$ ) con cualquiera de las dosis de $\mathrm{P}$ adicionadas, pero incrementándose el efecto residual al aumentar la dosis. Para sostener la productividad del sitio, los autores recomendaron emplear la dosis de $50 \mathrm{~kg} \mathrm{P} \mathrm{ha}^{-1}$ a la siembra y repetirla después del sexto año de cultivo sucesivo (Díaz-López et al., 2013).

La respuesta a la adición al $\mathrm{K}$ se ha observado en el 5 al $30 \%$ de los ensayos de campo en América Latina (Bartholomew, 1973) y en suelos con pH menor a 5,5 se ha encontrado mejorías con la aplicación de 1 a $2 \mathrm{t} \mathrm{ha}^{-1}$ de carbonato de calcio acompañado de 100-150 $\mathrm{kg} \mathrm{P}_{2} \mathrm{O}_{5}$ ha $^{-1}$ en Brasil (Bartholomew, 1973) y en Costa Rica (González \& González, 1981).

En el trópico estacionalmente seco de Costa Rica la información disponible sobre aspectos nutricionales para la producción de maíz es escaza. El rendimiento de grano en el trópico húmedo de Centroamérica varía entre 1 y $14 \mathrm{t} \mathrm{ha}^{-1}$ con un requerimiento de nutrientes totales $\left(\mathrm{kg} \mathrm{ha}^{-1}\right)$ de: 22 a 376 de N, 5 a 54 de P, 18 a 249 de K, 6 a 65 de Ca y 4 a 75 de $\mathrm{Mg}$ (Bertsch, 2009). Para obtener 6,25 tha ${ }^{-1}$ de grano con el híbrido de maíz HC-57 en la zona de Alajuela se reportaron requerimientos totales de nutrientes del orden de $\left(\mathrm{kg} \mathrm{ha}^{-1}\right): 111,2 \mathrm{de} \mathrm{N}, 18,7 \mathrm{de} \mathrm{P}, 140,2$ de K, 18,8 de Ca, 16,9 de Mg y 9,9 de S (Fallas et al., 2011). Sobre suelos de origen aluvial en la Zona Sur y suelos ácidos de la Zona Norte de Costa Rica, se encontraron rendimientos de grano de 2 a $4 \mathrm{t} \mathrm{ha}^{-1}$ con las variedades LD-8843 y J-Sáenz respectivamente, con la aplicación de ( $\left.\mathrm{kg} \mathrm{ha}^{-1}\right)$ : 100 de N, 60 de P, 40 de K, 20 de Mg y 20 de S (Bonilla, 2010a; 2010b).

Algunos materiales mejorados de maíz presentan a nivel de campo problemas en el recubrimiento total de la mazorca o poco desarrollo de la tusa, daño reconocido como mazorca expuesta, pues inhibe que la tusa recubra el $100 \%$ de los granos, la expone al ambiente y en ocasiones causa un crecimiento anormal en la mazorca (Ohio State University, 2017). Este daño ocurre principalmente entre los estados de desarrollo V5, V6, R1 (Ciampitti, 2014) y según Aldrich et al. (1986) se inicia en el estado R3, posterior a la etapa de polinización. El mal se considera como un desorden de crecimiento generado por problemas abióticos múltiples, entre ellos una mala nutrición de las plantaciones y una combinación de factores climáticos adversos como estrés por calor, poca disponibilidad de agua, temperaturas bajas y exceso de lluvia (Ciampitti, 2014; Nielsen, 2012). La exposición de las mazorcas en el campo, incentiva a los pájaros al consumo de los granos, lo cual reduce su calidad, incrementa las infecciones por hongos y mohos y pérdidas en la productividad, factores que potencian una caída en el precio del producto (Aldrich et al., 1986; Elmore et al., 2016). En Costa Rica no se han reportado casos de afectaciones de mazorca expuesta en maíz, aunque los productores en la zona de Guanacaste han observado el problema en campo que luego asocian a problemas de almacenamiento de las mazorcas, debido a una mayor incidencia de plagas y enfermedades.

El objetivo de este trabajo fue evaluar en la variedad de maíz J-Sáenz, el efecto de dosis crecientes de nitrógeno (N) y potasio $(\mathrm{K})$, en combinación con tres densidades de siembra.

\section{Materiales y métodos}

\section{Caracterización del sitio}

La investigación se realizó en la Finca Experimental de la Universidad de Costa Rica en el cantón de Santa Cruz, Guanacaste, durante el periodo marzo - julio del 2018. La finca se encuentra localizada a $10^{\circ} 17^{\prime} 6,24^{\prime \prime}$ latitud $\mathrm{N} \mathrm{y} 85^{\circ} 35^{\prime} 42,95^{\prime}$ " longitud $\mathrm{O}$, a una altitud aproximada de $54 \mathrm{msnm}$. El sitio presenta un clima estacionalmente 
seco, con precipitaciones promedio de $1834 \mathrm{~mm}_{\text {año }}{ }^{-1}$ entre los meses de abril - noviembre y seco entre diciembre - marzo y una temperatura promedio de $27,9^{\circ} \mathrm{C}$. Por sus condiciones climáticas y de relieve, los principales suelos de la finca son de textura arcillosa con características vérticas y se reconocen como Vertic Haplustalfs asociados a Typic Haplusterts y Typic Ustorthents (Guzmán, 1987; Salazar, 1991; Vega \& Salas, 2012). Los suelos presentan concentraciones disponibles de $\mathrm{Ca}, \mathrm{Mg}, \mathrm{K}, \mathrm{Cu} \mathrm{Fe}$ y $\mathrm{Mn}$, según los niveles críticos no tienen problemas de acidez intercambiable ni de salinidad (CE), su pH es óptimo para la absorción de nutrimentos por las plantas, el P presentó concentraciones muy bajas $\left(2 \mathrm{mg} \mathrm{l}^{-1}\right)$ y el Zn mostró valores cercanos al nivel crítico (Cuadro 1).

Cuadro 1. Contenido de nutrimentos en suelo utilizado para evaluar el efecto de tres dosis de $\mathrm{N}$ y K en combinación con tres densidades de siembra en maíz, en la Finca Experimental Santa Cruz, de la Universidad de Costa Rica, Guanacaste, Costa Rica. 2018.

Table 1. Nutrient content in soil used to evaluate the effect of three doses of $\mathrm{N}$ and $\mathrm{K}$ in combination with three sowing densities in corn at the Santa Cruz Experimental Farm, Universidad de Costa Rica, Guanacaste. 2018.

\begin{tabular}{|c|c|c|c|c|c|c|c|c|c|c|c|c|c|}
\hline \multirow{2}{*}{$\begin{array}{c}\text { KCl } \\
\text { Olsen }\end{array}$} & \multirow{2}{*}{$\begin{array}{c}\text { pH } \\
\mathbf{H}_{2} \mathrm{O}\end{array}$} & \multicolumn{5}{|c|}{$\operatorname{cmol}(+) \mathrm{l}^{-1}$} & \multirow[t]{2}{*}{$\% \mathrm{SA}$} & \multicolumn{5}{|c|}{$\mathrm{mg} \mathrm{l}^{-1}$} & \multirow{2}{*}{$\begin{array}{c}\begin{array}{c}\mathrm{mS} \\
\mathbf{c m}^{-1}\end{array} \\
\mathrm{CE}\end{array}$} \\
\hline & & acidez & $\mathbf{C a}$ & Mg & $\mathbf{K}$ & CICE & & $\mathbf{P}$ & $\mathrm{Zn}$ & $\mathrm{Cu}$ & $\mathrm{Fe}$ & Mn & \\
\hline $\mathrm{NC}$ & 5,5 & 0,5 & 4 & 1 & 0,2 & 5 & & 10 & 3 & 1 & 10 & 5 & 1,5 \\
\hline Suelo & 6,0 & 0,10 & 25,0 & 10,3 & 0,34 & 35,7 & 0,3 & 2 & 3,1 & 14 & 25 & 90 & 0,2 \\
\hline
\end{tabular}

Solución extractora KCl-Olsen modificado, Laboratorio Suelos y Foliares, Centro de Investigaciones Agronómicas, Universidad de Costa Rica; NC: nivel crítico para suelos en general según parámetros del laboratorio. CICE: Capacidad de intercambio de cationes efectiva: Acidez $+\mathrm{Ca}+\mathrm{Mg}+\mathrm{K} /$ Modified $\mathrm{KCl}-$ Olsen extractor solution, Soils and Foliar Laboratory, Agronomic research center, Universidad de Costa Rica. NC: Critical level for soils in general according to laboratory parameters. CICE: effective cation exchange capacity $=$ acidity $+\mathrm{Ca}+\mathrm{Mg}+\mathrm{K}$.

\section{Preparación del sitio y siembra}

El terreno se preparó en febrero de 2018 con dos pases de arado y dos de rastra para evitar la compactación superficial en un sitio en barbecho de varios años, homogéneo en cuanto al tipo de suelo y sin problemas de drenaje. Posteriormente, se cuadriculó el terreno y se marcó con estacas ochenta unidades experimentales con dimensiones de $6 \times 3 \mathrm{~m}\left(18 \mathrm{~m}^{2}\right.$ ). Se aplicó el herbicida preemergente (Pendimetalina) a razón de 2,5 $1 \mathrm{ha}^{-1}$ sobre el suelo y se sembró un mes después la variedad de maíz blanco (Zea mayz) J-Sáenz, esta es una planta de porte alto de hojas largas con una buena distribución en su filotaxia, presenta rendimientos entre los 2,9 a 3,5 t ha ${ }^{-1}$ según las dosis de nitrógeno en zonas rurales de Costa Rica (Bonilla, 2010b). Se trabajó con esta variedad debido a que es una de las más utilizadas por los productores en la zona de Guanacaste. Para esta investigación se evaluaron tres densidades de siembra, estas fueron de 50 000, 57143 y 66666 plantas ha $^{-1}$. Se utilizaron cintas de riego con una distancia de $15 \mathrm{~cm}$ entre goteros, colocando seis filas de cintas en cada unidad experimental, utilizadas únicamente en los momentos con poca disponibilidad de humedad en el suelo. Durante el desarrollo del cultivo se realizaron muestreos para verificar la presencia de plagas y para el combate de Spodoptera frugiperda Smith se utilizó Spinetoram (Solaris 6SC $®$ ) a una dosis de $0,0751 \mathrm{ha}^{-1}$.

\section{Tratamientos de fertilización}

Para establecer los ensayos de fertilización con $\mathrm{N}$ y K, el terreno preparado recibió una base de 60, 40, 54, 10 y $5 \mathrm{~kg} \mathrm{ha}^{-1}$ de $\mathrm{P}_{2} \mathrm{O}_{5}, \mathrm{MgO}, \mathrm{SO}_{4}$, Zn y B, con un fraccionamiento de $20 \%$ a los 10 días después de siembra (dds), $40 \%$ 
a los 30 dds y el restante $40 \%$ a los 45 dds. Se evaluaron los factores de densidad bajo el efecto de un nutrimento, para esto se establecieron dos experimentos simultáneos en forma separada con el fin de evaluar los factores $\mathrm{N} \mathrm{y}$ $\mathrm{K}$ por separados en función de la densidad de siembra. Posteriormente, el área se dividió en dos partes de manera que a la primera área (A) se le adicionó dosis de $\mathrm{N}$ a razón de: 100, 200, $300 \mathrm{~kg} \mathrm{ha}^{-1}$ con una base de $160 \mathrm{~kg} \mathrm{ha}^{-1} \mathrm{de}$ $\mathrm{K}_{2} \mathrm{O}$ y a la segunda área (B) dosis de $\mathrm{K}_{2} \mathrm{O}$ de 100,160 y $200 \mathrm{~kg} \mathrm{ha}^{-1}$ con una base de $300 \mathrm{~kg} \mathrm{ha}^{-1}$ de $\mathrm{N}$ (Cuadro 2). Estos rangos fueron seleccionados según los rangos encontrados en la literatura, se seleccionaron dosis intermedias de los nutrimentos y con base en la concentración química de nutrimentos del suelo. Las aplicaciones de fertilizantes se realizaron de forma manual con medidas específicas de las distintas fórmulas para cada tratamiento. Las dosis físicas de $\mathrm{N}$ y K se prepararon con las fuentes: nitrato de amonio (33,5-0 - 0), Kmag (0 - $0-22-18-22)$, fosfato diamónico (18 - 46 - 0), cloruro de potasio $\left(0-0\right.$ - 60), sulfato de zinc $\left(17 \% \mathrm{SO}_{4}\right.$ y $31 \% \mathrm{Zn}$ ) y ácido bórico (17,5\%). Para el cálculo del uso eficiente de nutrimentos se establecieron unidades experimentales sin fertilización de $\mathrm{N}$ y $\mathrm{K}$.

Cuadro 2. Descripción de los tratamientos de fertilización para evaluar el efecto de tres dosis de N y K en combinación con tres densidades de siembra en maíz en la Finca Experimental Santa Cruz, de la Universidad de Costa Rica, Guanacaste, Costa Rica. 2018.

Table 2. Fertilization treatments description to evaluate the effect of three doses of $\mathrm{N}$ and $\mathrm{K}$ in combination with three sowing densities in corn at the Santa Cruz Experimental Farm, of the Universidad de Costa Rica, Guanacaste. 2018.

\begin{tabular}{|c|c|c|c|c|c|c|c|c|}
\hline Tratamiento & Densidad (ha) & $\mathbf{N}$ & $\mathrm{K}_{2} \mathrm{O}$ & $\mathbf{P}_{2} \mathbf{O}_{5}$ & $\mathrm{MgO}$ & $\mathrm{SO}_{4}$ & $\mathbf{Z n}$ & B \\
\hline \multicolumn{9}{|c|}{ Área A } \\
\hline $\mathrm{N}$ absoluto & 50000 & 0 & 160 & & & & & \\
\hline $100 \mathrm{~N} 50$ & & 100 & 160 & & & & & \\
\hline $200 \mathrm{~N} 50$ & 50000 & 200 & 160 & & & & & \\
\hline $300 \mathrm{~N} 50$ & & 300 & 160 & 60 & 40 & 54 & 10 & 5 \\
\hline 100N 57 & & 100 & 160 & & & & & \\
\hline $200 \mathrm{~N} 57$ & 57143 & 200 & 160 & & & & & \\
\hline $300 \mathrm{~N} 57$ & & 300 & 160 & & & & & \\
\hline $100 \mathrm{~N} 66$ & & 100 & 160 & & & & & \\
\hline $200 \mathrm{~N} 66$ & 66667 & 200 & 160 & & & & & \\
\hline $300 \mathrm{~N} 66$ & & 300 & 160 & & & & & \\
\hline \multicolumn{9}{|c|}{ Área B } \\
\hline $\mathrm{K}$ absoluto & 50000 & 300 & 0 & & & & & \\
\hline 100K 50 & & 300 & 100 & & & & & \\
\hline $160 \mathrm{~K} 50$ & 50000 & 300 & 160 & & & & & \\
\hline 200K 50 & & 300 & 200 & & & & & \\
\hline 100K 57 & & 300 & 100 & & & & & \\
\hline 160K 57 & 57143 & 300 & 160 & 60 & 40 & 54 & 10 & 5 \\
\hline 200K 57 & & 300 & 200 & & & & & \\
\hline 100K 66 & & 300 & 100 & & & & & \\
\hline 160K 66 & 66667 & 300 & 160 & & & & & \\
\hline 200K 66 & & 300 & 200 & & & & & \\
\hline
\end{tabular}

\section{Variables evaluadas}

En la etapa fenológica R5 se evaluó la presencia del daño de mazorca expuesta contando el total de mazorcas presentes en cada unidad experimental y el número de mazorcas expuestas por tratamiento. En esta etapa fenológica 
se cosecharon cinco plantas de cada unidad experimental, se cuantificó el peso seco y se determinó la concentración foliar de N, P, Ca, Mg, K, S, Fe, Cu, Zn, Mn y B en el Laboratorio de Suelos y Foliar del Centro de Investigaciones Agronómicas de la Universidad de Costa Rica. Posteriormente, se cuantificó la absorción total de nutrimentos en las plantas por tratamiento experimental.

Se cosecharon todas las mazorcas de la parcela útil y se cuantificó el número de granos e hileras por mazorca, el rendimiento de grano al $14 \%$ de humedad y el peso de 1000 granos según la metodología del Centro Internacional de Mejoramiento de Maíz y Trigo (2012). Se cuantificó la absorción de nutrimentos y se calculó el uso eficiente de nutrientes, la eficiencia aparente de recuperación definida en función del incremento en peso $(\mathrm{kg})$ en la absorción sobre los $\mathrm{kg}$ de nutriente aplicado, la eficiencia interna de utilización que se traduce en los $\mathrm{kg}$ de rendimiento obtenido sobre los $\mathrm{kg}$ de nutriente absorbido; la eficiencia agronómica que es la cantidad en $\mathrm{kg}$ de incremento en el rendimiento sobre los $\mathrm{kg}$ de nutriente aplicado; la eficiencia fisiológica traducida en los $\mathrm{kg}$ de incremento en el rendimiento sobre los $\mathrm{kg}$ de nutriente absorbido; y el factor parcial de productividad que es la cantidad en $\mathrm{kg}$ de rendimiento sobre los $\mathrm{kg}$ de nutriente aplicado según la metodología descrita por el Instituto Internacional de Nutrición Vegetal (International Plant Nutrition Institute, 2007).

\section{Análisis de los datos}

Se realizó un análisis estadístico multicriterio para las variables de uso eficiente de nutrimentos según la metodología de Garbanzo \& Navarro (2015). Para las variables peso de 1000 granos, rendimiento al $14 \%$ de humedad y el índice de granos por metro cuadrado, se realizó un análisis de varianza con estructura factorial de tratamientos según el modelo especificado en la ecuación 1.

$$
y_{i j k}=\mu+\alpha_{i}+\beta_{j}+(\alpha \beta)_{i j}+\varepsilon_{i j k} \quad \text { Ecuación } 1
$$

Donde:

$y_{i j k}=$ es la respuesta de la k-ésima repetición del i-ésimo nivel de nitrógeno y el j-ésimo nivel de densidad.

$\mu=$ media general.

$\alpha_{i}=$ es el efecto fijo del i-ésimo nivel de nitrógeno. Con $\mathrm{i}=1, \ldots, 4$.

$\beta_{j}=$ es el efecto fijo del j-ésimo nivel de densidad. Con $\mathrm{i}=1, \ldots, 3$.

$(\alpha \beta)_{i j}=$ es el efecto fijo de la interacción entre las combinaciones de los niveles de nitrógeno y densidad.

$\varepsilon_{i j k}=$ error aleatorio asociado a cada observación ijk.

Todas las variables evaluadas presentaron heterocedasticidad, por lo que se incluyó una función del tipo varIdent en la matriz de varianza-covarianza del modelo de la ecuación 1. El término de interacción no fue significativo $(p>0,05)$ para ninguna de las variables, por lo que únicamente se analizó los efectos principales. Cuando fue pertinente, la separación de medias se hizo con la prueba DGC (Di-Rienzo et al., 2002), con un nivel de significancia $(\alpha)$ del 0,05 .

Para la variable porcentaje de mazorcas expuestas se realizó un análisis de devianza para un modelo lineal generalizado (MLG) con distribución binomial y función de enlace logit (Ecuación 2). Todos los análisis se realizaron con el software estadístico Infostat ${ }^{\circledR}$ (Di-Rienzo et al., 2018).

$$
\operatorname{logit}\left(E\left[y_{i j \mathrm{k}}\right]\right)=\mu+\alpha_{i}+\beta_{j}+(\alpha \beta)_{i j} \quad \text { Ecuación } 2
$$

Donde:

$y_{i j \mathrm{k}}=$ es la respuesta de la k-ésima repetición del i-ésimo nivel de nitrógeno y el j-ésimo nivel de densidad. 
$\mu=$ media general.

$\alpha_{i}=$ es el efecto fijo del i-ésimo nivel de nitrógeno. Con $\mathrm{i}=1, \ldots, 4$.

$\beta_{j}=$ es el efecto fijo del $\mathrm{j}$-ésimo nivel del factor B. Con $\mathrm{i}=1, \ldots, 3$.

$(\alpha \beta)_{i j}=$ es el efecto fijo de la interacción entre las combinaciones de los niveles de nitrógeno y densidad.

\section{Resultados}

El incremento de la densidad de siembra de la variedad de maíz J-Sáenz entre 50000 y 66667 plantas ha-1 $^{-1}$ elevó la producción de grano significativamente. Para las variables peso seco de 1000 granos, número de granos por $\mathrm{m}^{2}$ y rendimiento de grano a una humedad de $14 \%$, el aumento de la densidad de siembra causó un efecto altamente significativo $(\mathrm{p}<0,007)$, debido a un mayor número de granos por $\mathrm{m}^{2}$ (Cuadro 3$)$.

Cuadro 3. Valores e interacciones entre la densidad de siembra y dosis de $\mathrm{N}$ y $\mathrm{K}_{2} \mathrm{O}\left(\mathrm{kg} \mathrm{ha}^{-1}\right)$ encontrados sobre el rendimiento de grano (14 \% humedad) y el daño de mazorca expuesta (\%) en la variedad de maíz J-Saénz, en la Finca Experimental Santa Cruz, de la Universidad de Costa Rica, Guanacaste, Costa Rica. 2018.

Table 3. Values and interactions between sowing density and $\mathrm{N}$ and $\mathrm{K}_{2} \mathrm{O}$ doses $\left(\mathrm{kg} \mathrm{ha}^{-1}\right)$ found on grain yield (14\% moisture) and exposed ear damage (\%) in the J-Saénz corn variety in the Santa Cruz Experimental Farm, of the Universidad de Costa Rica, Guanacaste, Costa Rica. 2018.

\begin{tabular}{|c|c|c|c|c|c|c|c|c|}
\hline \multirow[t]{2}{*}{ Factores } & $\begin{array}{c}\text { Peso seco } \\
1000 \\
\text { granos }(\mathrm{g})\end{array}$ & $\begin{array}{c}\text { Número } \\
\text { granos } \\
\left(\mathbf{m}^{2}\right)\end{array}$ & $\begin{array}{c}\text { Rendimiento } \\
14 \% \\
\text { humedad (kg } \\
\left.\text { ha }^{-1}\right)\end{array}$ & $\begin{array}{c}\text { Daño } \\
\text { mazorca } \\
\text { expuesta } \\
(\%)\end{array}$ & $\begin{array}{l}\text { Peso seco } \\
1000 \\
\text { granos }(\mathrm{g})\end{array}$ & $\begin{array}{c}\text { Número } \\
\text { granos } \\
\left(\mathbf{m}^{2}\right)\end{array}$ & $\begin{array}{l}\text { Rendimiento } \\
14 \% \\
\text { humedad (kg } \\
\left.\text { ha }^{-1}\right)\end{array}$ & $\begin{array}{c}\text { Daño } \\
\text { mazorca } \\
\text { expuesta } \\
(\%)\end{array}$ \\
\hline & \multicolumn{4}{|c|}{ Efecto de la adición de $\mathrm{N}$} & \multicolumn{4}{|c|}{ Efecto de la adición de $\mathrm{K}_{2} \mathrm{O}$} \\
\hline Modelo & $<0,001$ & $<0,001$ & $<0,001$ & - & $<0,001$ & $<0,001$ & $<0,001$ & - \\
\hline Dosis & 0,1804 & 0,113 & 0,0631 & 0,4903 & 0,6223 & 0,9068 & 0,822 & 0,1667 \\
\hline Densidad & 0,0078 & 0,0001 & 0,0004 & 0,4305 & 0,3214 & 0,0025 & 0,0032 & $<0,001$ \\
\hline Dens. $\mathrm{x}$ dosis & 0,1995 & 0,217 & 0,2553 & 0,6217 & 0,6982 & 0,7111 & 0,7355 & 0,6555 \\
\hline Densidad & Medias & Medias & & & & & & \\
\hline 50000 & $231^{a^{*}}$ & $845^{c}$ & $2240^{b}$ & $4,3^{\text {a }}$ & $228^{\text {a }}$ & $1032^{b}$ & $2713^{b}$ & $5,8^{\mathrm{b}}$ \\
\hline 57143 & $226^{\mathrm{a}}$ & $1265^{b}$ & $3320^{\text {a }}$ & $2,4^{\text {a }}$ & $226^{\mathrm{a}}$ & $1367^{\text {a }}$ & $3864^{\text {a }}$ & $4,1^{c}$ \\
\hline 66667 & $212^{b}$ & $1618^{a}$ & $4015^{a}$ & $1,7^{\text {a }}$ & $224^{a}$ & $1488^{a}$ & $3624^{a}$ & $5,9^{\text {a }}$ \\
\hline
\end{tabular}

* Medias seguidas por una misma letra no son significativas $(\mathrm{p}>0,05)$ según prueba DGC $/ *$ Means followed by the same letter are not significant $(\mathrm{p}<0.05)$ according to the DGC test.

En el experimento con dosis crecientes de K hubo un efecto significativo para el factor densidad de siembra. Esta se encontró sobre las variables de rendimiento, a excepción de la variable peso seco de 1000 granos. El mayor número de granos por $\mathrm{m}^{2}$ y del rendimiento total se obtuvo con las densidades de 57143 y 66667 plantas ha $^{-1}$. La interacción densidad de siembra y dosis de $\mathrm{N}$ no afectaron la variable mazorca expuesta, aunque con el incremento en la dosis de $\mathrm{K}$ los mayores porcentajes de esta variable se dieron con las densidades de siembra de 50000 y 66 667 plantas ha-1. $^{-1}$

La mayor eficiencia en el uso de $\mathrm{N}$ y K se encontró con la aplicación de $100 \mathrm{~kg}$ de cada elemento a la densidad de 57000 plantas ha ${ }^{-1}$. El mayor índice multicriterio se logró con la adición de dosis bajas de $\mathrm{N}$ y la densidad 
de 57143 plantas ha-1 (Cuadro 4). La aplicación de K causó un efecto similar al de la adición de N; así para las variables eficiencia agronómica (EA) y factor parcial de productividad (FPP), los mayores índices de rendimiento se obtuvieron con dosis bajas de $\mathrm{N}$ y una densidad de 57143 plantas ha $^{-1}$, efecto similar al encontrado para FPP con dosis bajas de $\mathrm{K}$ a las mismas densidades.

Cuadro 4. Índices agronómicos de eficiencia de uso de N y K sobre de rendimiento de grano (14 \% humedad) de la variedad de maíz J. Saénz, bajo tres densidades de siembra y dosis de N y K en la Finca Experimental Santa Cruz, de la Universidad de Costa Rica, Guanacaste, Costa Rica. 2018.

Table 4. Agronomic indices of efficiency of use of $\mathrm{N}$ on grain yield (14\% moisture) of the J-Saénz corn variety under three planting densities and doses of $\mathrm{N}$ and $\mathrm{K}$ in the Santa Cruz Experimental Farm, of the Universidad de Costa Rica, Guanacaste, Costa Rica. 2018.

\begin{tabular}{lcccccc}
\hline Tratamiento & ER* & EI* & $\begin{array}{c}\text { EA }\left(\mathbf{k g ~ k g}^{-1}\right)^{*} \\
\mathbf{N}\end{array}$ & $\mathbf{E F}^{*}$ & FPP* & $\begin{array}{c}\text { Índice } \\
\text { multicriterio }\end{array}$ \\
\hline 100N 50 & 1,05 & 16,3 & 12,0 & $35,0^{\mathrm{a}}$ & 21,0 & 76 \\
200N 50 & 0,91 & 10,3 & 8,0 & $8,5^{\mathrm{a}}$ & 12,5 & 43 \\
300N 50 & 0,55 & 10,0 & 4,3 & $8,3^{\mathrm{a}}$ & 7,0 & 31 \\
100N 57 & 1,17 & 17,5 & 22,5 & $19,5^{\mathrm{a}}$ & 31,3 & 86 \\
200N 57 & 1,02 & 11,8 & 11,3 & $11,3^{\mathrm{a}}$ & 15,5 & 53 \\
300N 57 & 0,64 & 16,5 & 10,0 & $18,3^{\mathrm{a}}$ & 12,5 & 54 \\
100N 66 & 1,60 & 13,5 & 20,8 & $13,8^{\mathrm{a}}$ & 29,5 & 81 \\
200N 66 & 1,05 & 15,8 & 16,3 & $16,0^{\mathrm{a}}$ & 20,3 & 68 \\
300N 66 & 0,83 & 16,8 & 14,0 & $17,3^{\mathrm{a}}$ & 17,0 & 63 \\
\hline & & & & & 44 \\
\hline 100K 50 & 0,23 & 19,7 & 8,0 & $2,93^{\mathrm{a}}$ & 33,2 & 35 \\
160K 50 & 0,05 & 16,5 & 3,4 & $25,5^{\mathrm{ab}}$ & 19,2 & 47 \\
200K 50 & 0,15 & 16,0 & 4,2 & $55,8^{\mathrm{b}}$ & 16,8 & 87 \\
100K 57 & 0,40 & 21,4 & 21,2 & $55,2^{\mathrm{b}}$ & 46,5 & 52 \\
160K 57 & 0,10 & 25,7 & 13,3 & $13,0^{\mathrm{a}}$ & 29,1 & 56 \\
200K 57 & 0,13 & 23,0 & 10,4 & $41,7^{\mathrm{b}}$ & 23,1 & 73 \\
100K 66 & 0,78 & 16,0 & 13,7 & $29,3^{\mathrm{b}}$ & 39,0 & 71 \\
160K 66 & 0,30 & 21,3 & 14,7 & $56,2^{\mathrm{b}}$ & 30,5 & 50 \\
\hline 200K 66 & 0,45 & 16,2 & 8,73 & $22,8^{\mathrm{ab}}$ & 21,4 & \\
\hline
\end{tabular}

*ER: eficiencia aparente de recuperación; EI: eficiencia interna de utilización; EA: eficiencia agronómica; EF: eficiencia fisiológica; FPP: factor parcial de productividad / * ER: Recovery efficiency; EI: Utilization eficiency; EA: Agronomic effectiveness; EF: Physiological effectiveness; FPP: Grain yield efficiency.

Se encontró que las plantas de maíz respondieron al incremento en las dosis de N, mientras que la dosificación de $\mathrm{K}$ no causó diferencias en la absorción de estos nutrimentos (Cuadro 5). Al analizar el factor densidad presentó significancia en las dosis de $\mathrm{N}$ y $\mathrm{K}$, mientras que la dosis en función de factor solo fue significativa en las aplicaciones de $\mathrm{N}$ para la absorción de los nutrimentos. La interacción evaluada en el modelo para los factores dosis y densidad no mostraron un efecto significativo en la absorción de los nutrimentos. 
Cuadro 5. Absorción de nutrimentos en maíz variedad J-Saénz en el estado fenológico R5 bajo tres densidades de siembra en la Finca Experimental Santa Cruz, de la Universidad de Costa Rica, Guanacaste, Costa Rica. 2018.

Table 5. Nutrient uptake at R5 phenological stage in J-Saénz corn variety under three sowing densities at the Santa Cruz Experimental Farm, of the Universidad de Costa Rica, Costa Rica. 2018.

\begin{tabular}{|c|c|c|c|c|c|c|c|}
\hline Tratamientos & $\mathbf{N}$ & $\mathbf{P}$ & $\mathbf{K}$ & Mg & $\mathbf{S}$ & Zn & B \\
\hline Fertilización con $\mathbf{N}$ & & & kg ha $^{-1}$ & & & \multicolumn{2}{|c|}{$\mathrm{mg} \mathrm{kg}^{-1}$} \\
\hline $100 \mathrm{~N} 50$ & 165 & 32 & 153 & 32 & 13 & 547 & 72 \\
\hline $200 \mathrm{~N} 50$ & 242 & 49 & 201 & 38 & 19 & 688 & 66 \\
\hline $300 \mathrm{~N} 50$ & 223 & 32 & 167 & 33 & 14 & 519 & 48 \\
\hline $100 \mathrm{~N} 57$ & 175 & 31 & 156 & 31 & 14 & 560 & 108 \\
\hline 200 N 57 & 263 & 48 & 217 & 46 & 22 & 844 & 80 \\
\hline 300 N 57 & 250 & 37 & 176 & 41 & 17 & 730 & 64 \\
\hline $100 \mathrm{~N} 66$ & 219 & 43 & 169 & 43 & 17 & 780 & 104 \\
\hline $200 N 66$ & 268 & 50 & 188 & 44 & 20 & 704 & 91 \\
\hline $300 N 66$ & 309 & 55 & 243 & 51 & 23 & 910 & 81 \\
\hline Factores & \multicolumn{7}{|c|}{ p. value } \\
\hline Modelo & $<0,01$ & $<0,01$ & $<0,01$ & $<0,01$ & $<0,01$ & $<0,01$ & $<0,01$ \\
\hline Densidad & 0,02 & 0,01 & 0,05 & $<0,01$ & $<0,01$ & $<0,01$ & $<0,01$ \\
\hline Dosis & $<0,01$ & $<0,01$ & $<0,01$ & $<0,01$ & $<0,01$ & $<0,01$ & $<0,01$ \\
\hline Densidad $\mathrm{x}$ dosis & 0,67 & 0,39 & 0,20 & 0,12 & 0,12 & 0,05 & 0,01 \\
\hline Fertilización con $K_{2} \mathbf{0}$ & \multicolumn{5}{|c|}{ kg ha $^{-1}$} & \multicolumn{2}{|c|}{$\mathrm{mg} \mathrm{kg}^{-1}$} \\
\hline $100 \mathrm{~K} 50$ & 256 & 37 & 170 & 38 & 19 & 576 & 59 \\
\hline $160 \mathrm{~K} 50$ & 212 & 33 & 155 & 38 & 15 & 780 & 71 \\
\hline $200 \mathrm{~K} 50$ & 288 & 45 & 173 & 50 & 21 & 690 & 91 \\
\hline $100 \mathrm{~K} 57$ & 276 & 53 & 184 & 48 & 21 & 748 & 90 \\
\hline 160K 57 & 249 & 50 & 163 & 47 & 17 & 780 & 84 \\
\hline 200K 57 & 242 & 40 & 172 & 39 & 16 & 570 & 80 \\
\hline $100 \mathrm{~K} 66$ & 320 & 66 & 213 & 60 & 21 & 932 & 119 \\
\hline $160 \mathrm{~K} 66$ & 288 & 53 & 190 & 43 & 19 & 755 & 92 \\
\hline $200 \mathrm{~K} 66$ & 304 & 65 & 225 & 54 & 22 & 911 & 118 \\
\hline Factores & \multicolumn{7}{|c|}{ p. value } \\
\hline Modelo & $<0,01$ & $<0,01$ & $<0,01$ & $<0,01$ & $<0,01$ & $<0,01$ & $<0,01$ \\
\hline Densidad & 0,09 & 0,01 & 0,01 & 0,03 & 0,20 & 0,01 & $<0,01$ \\
\hline Dosis & 0,47 & 0,60 & 0,48 & 0,31 & 0,22 & 0,58 & 0,01 \\
\hline Densidad $\mathrm{x}$ dosis & 0,94 & 0,82 & 0,99 & 0,28 & 0,73 & 0,47 & 0,17 \\
\hline
\end{tabular}

\section{Discusión}

La productividad de grano obtenida respondió al aumento en la densidad de siembra, tanto para las dosis de $\mathrm{N}$ como para las de K. Este efecto se alcanzó por un mayor número de granos por metro cuadrado y no por la biomasa del grano, es probable que la asimilación fuera más eficiente en densidades altas por la arquitectura que presentó la planta. Las densidades de 57143 y 66667 plantas ha $^{-1}$ bajo fertilización nitrogenada, mostraron una producción 
de 3320 y $4015 \mathrm{~kg} \mathrm{ha}^{-1}$ de grano a un $14 \%$ de humedad, sin diferencias significativas; esto concuerda con Bonilla (2010b), que encontró un rendimiento de $3680 \mathrm{~kg} \mathrm{ha}^{-1}$ a una densidad de 53333 plantas ha ${ }^{-1}$ para esta variedad en la zona central sur de Costa Rica. Sin embargo, pese a que el autor no reportó el porcentaje de humedad del grano, sí demostró una mayor productividad en el trópico húmedo que el seco. El rendimiento no presentó estadísticamente una relación directa con las dosificaciones de $\mathrm{N} \mathrm{y}_{2} \mathrm{O}$, es probable que el suelo aportara una cantidad importante de los nutrimentos de forma soluble para las plantas (Cuadro 1) lo que permitió que el cultivo mostrara suficiente de absorción en las dosificaciones de $100 \mathrm{~kg} \mathrm{ha}^{-1}$ de $\mathrm{N} \mathrm{y} \mathrm{K}_{2} \mathrm{O}$.

El daño de mazorca expuesta no fue generalizado y contribuyó en un daño inferior al 6,5\% del total de las mazorcas evaluadas, efecto no significativo en comparación a otros daños causados por plagas o enfermedades en el sitio. Las densidades y las dosificaciones con $\mathrm{N}$ no mostraron efectos significativos en la mazorca expuesta, sin embargo, al evaluar las densidades de siembra con distintas dosis de $\mathrm{K}_{2} \mathrm{O}$, este elemento mostró un efecto significativo entre las densidades. En este experimento se encontró presencia no significativa de la mazorca expuesta en todos los tratamientos, no obstante, se encontró la tendencia que al aumentar las dosis de $\mathrm{N}$ y K se presentó mayor porcentaje de mazorcas expuestas. Es posible que estos efectos sean producto de un desorden fisiológico potencializado por altas dosis de fertilizantes (Ciampitti, 2014).

Las densidades de siembra y las dosis de $\mathrm{N}$ afectaron la absorción de $\mathrm{N}, \mathrm{P}, \mathrm{K}, \mathrm{Mg}, \mathrm{S}, \mathrm{Zn}$ y B. La dosis de $200 \mathrm{~kg}$ de $\mathrm{N} \mathrm{ha}^{-1}$ en densidades de 50000 y 57143 planta ha $^{-1}$, mostró la mayor absorción, es posible que estas acumulaciones se presentaron en función de la biomasa que alcanzaron las plantas de maíz, debido a que este incremento es correlativo con $\mathrm{P}, \mathrm{K}, \mathrm{Mg}, \mathrm{S}$ y Zn. Este mismo efecto se encontró en la dosis de $300 \mathrm{~kg}$ de $\mathrm{N} \mathrm{ha}^{-1}$ en la densidad de 66667 plantas ha-1 $^{-1}$, no obstante, al analizar las acumulaciones de B en la planta, se presentó una mayor acumulación con las dosis más bajas de $\mathrm{N}$. La mayor acumulación de $\mathrm{N}$ en el sistema (309 kg ha-1) se presentó en plantas sembradas a las densidades más elevadas con una dosis de $300 \mathrm{~kg} \mathrm{~N} \mathrm{ha}^{-1}$.

Las dosis de $\mathrm{K}$ no mostraron un efecto significativo en la acumulación de nutrimentos, sin embargo, mostraron una mayor acumulación cuando aumentó la densidad. Este efecto se debe a que existió mayor cantidad de biomasa, lo que aumenta la acumulación de nutrimentos por ha. El efecto también se vio marcado para los tratamientos que tuvieron mayor acumulación de $\mathrm{N}, \mathrm{P}, \mathrm{Mg}, \mathrm{S}$ y B. El Zn mostró un comportamiento distinto porque presentó concentraciones más altas en dosis intermedias $\left(160 \mathrm{~kg} \mathrm{~K}_{2} \mathrm{O}\right.$ ha-1 $)$ de P. La absorción máxima que presentó el $\mathrm{K}$ en maíz fue de $225 \mathrm{~kg} \mathrm{ha}^{-1}$ en altas densidades.

La dosis de $100 \mathrm{~kg}_{\text {de }} \mathrm{N}_{\text {ha }}{ }^{-1}$ mostró el uso más eficiente del nutrimento para una densidad de siembra de 57143 plantas ha ${ }^{-1}$ según el análisis multicriterio. La eficiencia aparente de recuperación de $\mathrm{N} \mathrm{y} \mathrm{K}$ determinaron que los tratamientos con las dosis más bajas en las tres densidades lograron incrementar la absorción de ambos elementos en la planta. Es posible que este efecto fuera producto de que el suelo tuviera altas concentraciones de los nutrimentos en forma disponible (Cuadro 1), esto hizo que gran parte de la absorción por la planta fuera suministrada por el suelo y no por las dosis de nutrimentos aplicadas, e influyó en que la eficiencia aparente de recuperación fuera baja. La eficiencia interna de utilización mostró que, según el rendimiento alcanzado para cada tratamiento, las dosis más bajas de fertilizante nitrogenado con densidades bajas a medias de siembra, fueron eficientes, esto quiere decir que por cada kg de $\mathrm{N}$ absorbido se produjo $17 \mathrm{~kg}$ en el rendimiento. Los rangos óptimos para el cultivo de maíz se encuentran entre 30 - $90 \mathrm{~kg} \mathrm{~kg}^{-1}$ de N (International Plant Nutrition Institute, 2007), los cuales están por arriba del alcanzado por el experimento $\left(17,5 \mathrm{~kg} \mathrm{~kg}^{-1}\right)$. Por otra parte, la eficiencia agronómica mostró que las dosis bajas de $\mathrm{N}$ presentaron las mayores eficiencias en las distintas densidades $\left(12-22,5 \mathrm{~kg} \mathrm{~kg}^{-1}\right)$. Este mismo comportamiento se observó para la eficiencia fisiológica y factor parcial de productividad.

Para este estudio, el análisis multicriterio fue una buena herramienta para seleccionar la mejor dosis y densidad. Los tratamientos con mayores índices concordaron con lo encontrado en el rendimiento (Cuadro 3). La metodología empleada para el cálculo de los índices relacionó criterios de especialistas en el campo y datos cuantitativos encontrados para este trabajo (Garbanzo \& Navarro, 2015). 


\section{Conclusiones}

El mayor rendimiento y uso eficiente de nutrimentos se dio con la densidad de siembra de 57143 plantas ha-1,

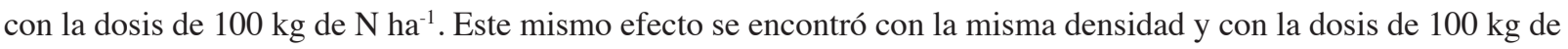
$\mathrm{K}_{2} \mathrm{O} \mathrm{ha}^{-1}$, debido a que no existieron diferencias significativas al aumentar las dosis de aplicación de cada elemento en ambos experimentos.

Hubo presencia de mazorca expuesta pero sin relación con las dosis de nutrimentos, ya que el porcentaje de daño causado por este desorden fisiológico no superó el 6,5 \% en las plantación de maíz blanco.

\section{Referencias}

Aguilar, C., Salvador, J., \& Aguilar, I. (2015). Análisis de crecimiento y rendimiento de maíz en clima cálido en función del genotipo, biofertilizante y nitrógeno. Terra Latinoamericana, 33(1), 51-62.

Aldrich, S., Scott, W., \& Hoeft, R. (1986). Modern corn production. A and L Publications.

Alvarado, A., Bertsch, F., Bornemisza, E., Cabalceta, G., Forsyth, W., Henríquez, C., Mata, R., Molina, E., \& Salas, R. (2001). Suelos derivados de cenizas volcánicas (Andisoles) de Costa Rica. Asociación Costarricense de la Ciencia del Suelo.

Bartholomew, W. (1973). Un resumen de las investigaciones edafológicas en américa latina tropical. North Carolina State University.

Bertsch, F. (2009). Absorción de nutrimentos por los cultivos. Asociación Costarricense de la Ciencia del Suelo.

Bonilla, N. (2010a). Análisis de correlación y regresión para la determinación del nitrógeno en maíz. Alcances Tecnológicos, 8(1), 5-14. https://doi.org/10.35486/at.v8i1.74

Bonilla, N. (2010b). Fertilización nitrogenada de variedades de maíz en dos localidades maiceras de Costa Rica. Alcances Tecnológicos, 8(1), 39-44. https://doi.org/10.35486/at.v8i1.77

Bonilla, N., \& Meléndez, W. (2005). Curso producción de semillas de maíz. Ministerio de Agricultura y Ganadería.

Bonilla, N., Vargas, A., Acuña, A., León, R., Guzmán, P., Rodríguez, C., \& Carrillo, O. (2008). Cultivo de maíz (Zea mays). Manual de recomendaciones técnicas del cultivo de maíz. Instituto Nacional de Transferencia Agropecuaria.

Castillo, R., \& Bird, R. (2013). Caracterización del cultivo de maíz en Nicaragua: un análisis de varianza de los determinantes del rendimiento. Banco Central de Nicaragua.

Ciampitti, I. (2014). Abnormal corn ears. Kansas State University Agricultural Experiment Station and Cooperative Extension Service.

Centro Internacional de Mejoramiento de Maíz y Trigo. (2012). Manual de determinación de rendimiento. Centro Internacional de Mejoramiento de Maíz y Trigo.

Di-Rienzo, J. A., Casanoves, F., Balzarini, M. G., González, L., Tablada, M., \& Robledo, C. W. (2018). Software estadístico. Grupo InfoStat. Recuperado el 15 de julio, 2019 de http://www.infostat.com.ar

Di-Rienzo, J. A., Guzmán, A. W., \& Casanoves, F. (2002). A multiple-comparisons method based on the distribution of the root node distance of a binary tree. Journal of Agricultural, Biological, and Environmental Statatistics, 7(2),129-142. https://doi.org/10.1198/10857110260141193 
Díaz-López, E., Loeza-Cortes, J., Campos-Pastelín, J., Morales-Rosales, E., Domínguez-López, A., \& Franco-Mora, O. (2013). Eficiencia en el uso de la radiación, tasa de asimilación neta e integral térmica en función del fósforo en maíz (Zea mays L.). Agrociencia, 47(2), 135-146.

Elmore, R., Rees, J., McMechan, J., Jackson, T., \& Hoegemeyer, T. (2016). Corn ear formation issues likely correlated whit the loss of the primary ear node. University of Nebraska-Lincoln. Retrieved September 27, 2019, from http://cropwatch. unl.edu/2016/corn-ear-formation-issues-likely-correlated-loss-primary-ear-node

Fallas, R., Bertsch, F., Echandi, C., \& Henríquez, C. (2011). Caracterización del desarrollo y absorción de nutrimentos del híbrido de maíz HC-57. Agronomía Costarricense, 35(2), 33-47. https://doi.org/10.15517/RAC.V35I2.6677

Garbanzo, G., \& Navarro, J. (2015). Análisis multicriterio de variables químicas, físicas y biológicas en 10 mezclas de sustratos hortícolas en Guanacaste, Costa Rica. InterSedes, 16(33), 71-81. https://doi.org/10.15517/ISUCR.V16I33.19026

González, L., \& González, M. (1981). Neutralización del aluminio cambiable en diez suelos de San Carlos y Sarapiquí, Costa Rica. Agronomía Costarricense, 5, 37-48.

Gordon, R., González, A., \& Franco, J. (2000). Manejo de la fertilización nitrogenada en el cultivo de maíz, Azuero, Panamá, 1996-1998. Ciencia Agropecuaria, 10, 123-134.

Gordon-Mendoza, R., Franco-Barrera, J., \& Villarreal-Núñez, J. (2016). Manejo de la fertilización fosforada en el cultivo de maíz, El Ejido, Panamá 2004-2013. Agronomía Mesoamericana, 28(1), 95-108. https://doi.org/10.15517/ am.v27i1.21889

Guzmán, D. (1987). Estudio detallado de suelos y clasificación de uso y aptitud para riego de las tierras de la Finca Experimental Santa Cruz (Tesis de Licenciatura, no publicada) Universidad de Costa Rica.

International Plant Nutrition Institute. (2007). Consideraciones en el uso eficiente de nutrientes. Informaciones Agronómicas. International Plant Nutrition Institute.

Nielsen, R. (2012). Short husks and exposed ears. Purdue University. Retrieved December 4, 2019, from https://www.agry. purdue.edu/ext/corn/news/timeless/EarHusks.html

Ohio State University. 2017. Troubleshooting abnormal corns ears. Exposed ear tips in corn. Retrieved December 4, 2019, from http://u.osu.edu/mastercorn/exposed-ear-tips/

Sáenz, A. (1970). Historia agrícola de Costa Rica. Universidad de Costa Rica.

Salas, C. (1971). Algunas recomendaciones para producir más maíz. Ministerio de Agricultura y Ganadería.

Salazar, N. (1991). Fertilidad de suelos de la Finca Experimental de Santa Cruz (prueba de invernadero) Guanacaste, Costa Rica (Tesis de Licenciatura, no publicada). Universidad de Costa Rica.

Salas, C., \& Bonilla, N. (1961). Ensayos uniformes de fertilización del maíz en Costa Rica. Benjamín Franklin.

Vega, E., \& Salas, R. (2012). Curvas de absorción de nutrientes bajo dos métodos de fertilización en sandia, en Guanacaste, Costa Rica. Revista electrónica de las sedes regionales de la Universidad de Costa Rica. InterSedes, 13(26), 21-42. https://revistas.ucr.ac.cr/index.php/intersedes/article/view/2988 